\title{
BMJ Pulmonary metastasectomy in colorectal open cancer: a prospective study of demography and clinical characteristics of 543 patients in the Spanish colorectal metastasectomy registry (GECMP-CCR)
}

To cite: Embún $\mathrm{R}$,

Fiorentino F, Treasure $\mathrm{T}$, et al. Pulmonary metastasectomy in colorectal cancer: a prospective study of demography and clinical characteristics of 543 patients in the Spanish colorectal metastasectomy registry (GECMP-CCR). BMJ Open 2013;3:e002787. doi:10.1136/bmjopen-2013002787

- Prepublication history and additional material for this paper are available online. To view these files please visit the journal online (http://dx.doi.org/10.1136/ bmjopen-2013-002787).

Received 25 February 2013 Accepted 9 April 2013

This final article is available for use under the terms of the Creative Commons Attribution Non-Commercial 2.0 Licence; see http://bmjopen.bmj.com

For numbered affiliations see end of article.

Correspondence to Professor Tom Treasure; tom.treasure@gmail.com

\section{ABSTRACT}

Objectives: To capture an accurate contemporary description of the practice of pulmonary metastasectomy for colorectal carcinoma in one national healthcare system.

Design: A national registry set up in Spain by Grupo Español de Cirugía Metástasis Pulmonares de Carcinoma Colo-Rectal (GECMP-CCR).

Setting: 32 Spanish thoracic units.

Participants: All patients with one or more histologically proven lung metastasis removed by surgery between March 2008 and February 2010.

Interventions: Pulmonary metastasectomy for one or more pulmonary nodules proven to be metastatic colorectal carcinoma.

Primary and secondary outcome measures: The age and sex of the patients having this surgery were recorded with the number of metastases removed, the interval between the primary colorectal cancer operation and the pulmonary metastasectomy, and the carcinoembryonic antigen level. Also recorded were the practices with respect to mediastinal lymphadenopathy and coexisting liver metastases.

Results: Data were available on 543 patients from 32 units (6-43/unit). They were aged 32-88 (mean 65) years, and $65 \%$ were men. In $55 \%$ of patients, there was a solitary metastasis. The median interval between the primary cancer resection and metastasectomy was 28 months and the serum carcinoembryonic antigen was low/normal in the majority. Liver metastatic disease was present in $29 \%$ of patients at some point prior to pulmonary metastasectomy. Mediastinal lymphadenectomy varied from $9 \%$ to $100 \%$ of patients. Conclusions: The data represent a prospective comprehensive national data collection on pulmonary metastasectomy. The practice is more conservative than the impression gained when members of the European Society of Thoracic Surgeons were surveyed in 2006/ 2007 but is more inclusive than would be recommended on the basis of recent outcome analyses. Further

\section{ARTICLE SUMMARY}

Article focus

- Surgical excision of lung metastases from colorectal cancer has been practiced with increasing frequency for 50 years.

- Belief in effectiveness is based on clinical impression and retrospective analysis of over 90 selected case series; there have been no controlled studies.

- A European Society of Thoracic Surgeons working group found the evidence insufficient to make recommendations.

- This study provides a hitherto missing element which is a record at a population level of the selective nature of this practice and the characteristics of patients having this surgery.

Key messages

- A prospective registry captured data on patients having pulmonary metastasectomy among an estimated $60 \%$ of the Spanish population.

- Patients having this surgery are highly selected representing fewer than 1 in 20 patients with metastatic colorectal cancer. Of them, $55 \%$ had a solitary metastasis, and in $36 \%$ the interval between primary surgery and metastasectomy was more than 3 years, features which are likely to be associated with a good natural history and are not the typical pattern of advanced colorectal cancer.

- Many patients operated on had three or more metastases resected (25\%) and/or metastasectomy within 1 year $(17 \%)$ and on existing evidence, these can be expected to have early recurrence and poor survival.

analyses on the morbidity associated with this surgery and the correlation between imaging studies and pathological findings are being published separately by GECMP-CCR. 


\section{ARTICLE SUMMARY}

Strengths and limitations of this study

- The strength of the study is that it captures prospectively the number and nature of patients in a representative national practice during a 2-year period.

- The data are limited to patients who had metastasectomy for a proven colorectal metastasis.

- This is an observational study of practice; survival among these patients will be presented in a future publication.

\section{INTRODUCTION}

Colorectal carcinoma is the commonest cancer for which pulmonary metastasectomy is undertaken. Pulmonary metastasectomy for colorectal cancer is widely practiced and is included in the UK's National Institute for Health and Clinical Excellence guidance. ${ }^{1} 2$ Belief in its effectiveness in improving survival relies on more than 90 surgical follow-up studies ${ }^{3}$ going back to the $1960 \mathrm{~s}^{4}$ (figure 1). Several systematic reviews ${ }^{3-7}$ have been published, the most recent in 2013 summing up the available evidence as follows: "There were no prospective randomized study, and most articles reported a single-center experience of fewer than 80 patients operated during a time period of 15-20 years." ${ }^{3}$ While surgical removal of lung metastases is widely accepted to be beneficial 'in selected patients', it was argued more than 30 years ago that the perception of benefit may arise from the selection of favourable cases for surgery rather than a real benefit attributable to surgery. ${ }^{8}$

The European Society of Thoracic Surgeons (ESTS), aware that the practice of pulmonary metastasectomy was increasing, initiated in 2006 the Lung Metastasectomy Project. Its first product was a survey of the opinions and beliefs of its members. The data suggested widespread, liberal and uncontrolled use of this surgery in European practice. ${ }^{9}$ Most responding surgeons believed that there was no defined limit to the number of metastases that should be removed, over $90 \%$ were prepared to operate for metastases appearing within a year of primary resection, and three quarters were prepared to remove synchronous pulmonary metastases. This was in contrast to the then published evidence on practice. In the largest of the systematic reviews, including data on 3504 patients, $60 \%$ of operations were for solitary metastases, the average interval between primary surgery and metastasectomy was 36 months and $60 \%$ of patients were dead within 5 years. ${ }^{4}$ After a comprehensive review of the published evidence, the leaders of the ESTS Project reported "that although there was great experience in performing this surgery, the belief in its benefit relied on clinical case series and registry reports. Evidence fell well short of Evidence Based Medicine standards and robust guidance could not be produced on this basis." ${ }^{\circ 1}$ It was clear to the ESTS project leaders that better evidence was needed. ${ }^{10}$ As a result, a randomised trial, PulMiCC (Pulmonary Metastasectomy in Colorectal Cancer), is recruiting internationally in Europe. $^{11}$

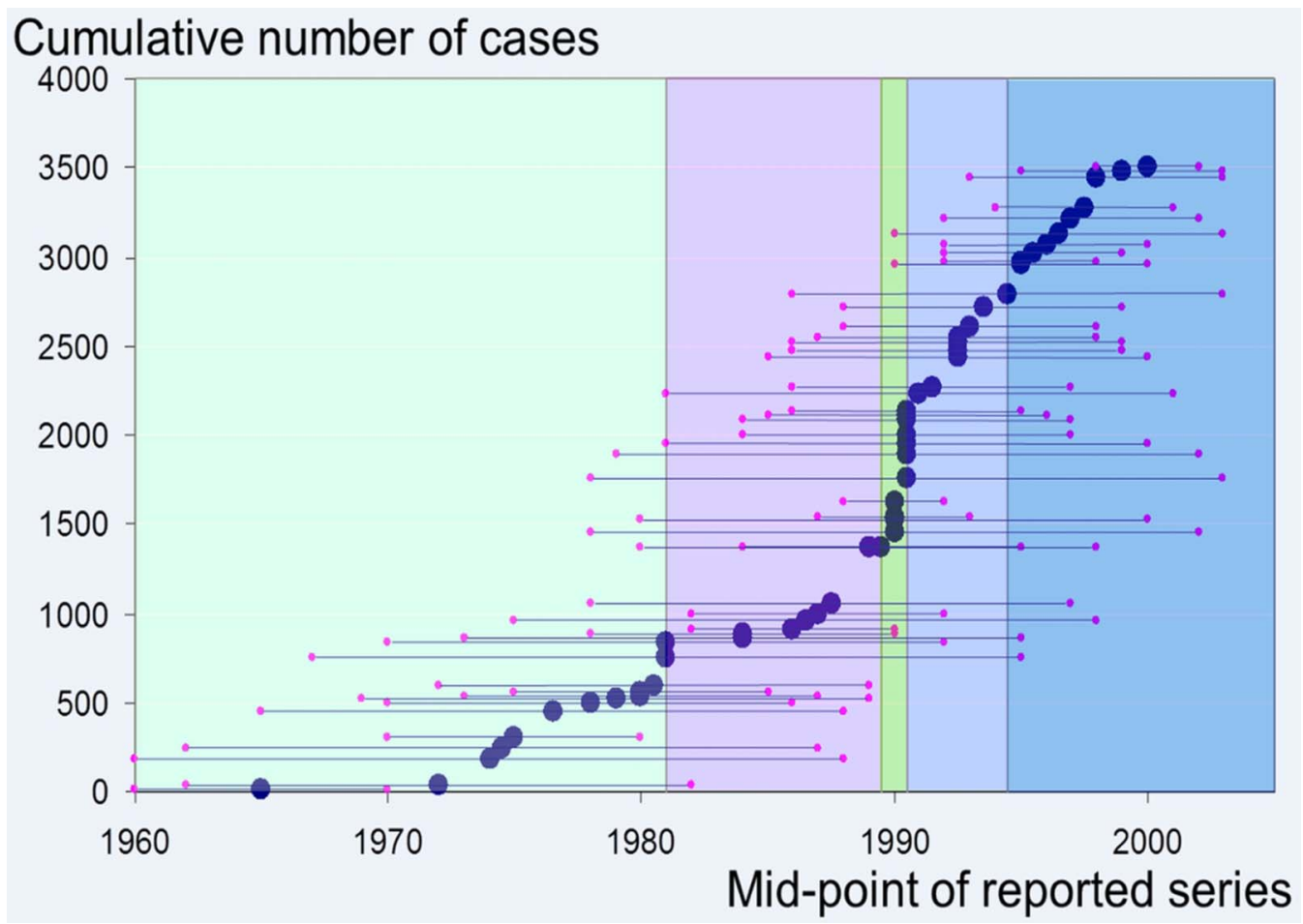

Figure 1 The start mid point and end dates of 51 reported series in the systematic review by Fiorentino et al. ${ }^{4}$ The vertical coloured bands delineate quintiles in accrual of the 3504 patients in the quantitative synthesis. 
Not only is there a lack of evidence after 50 years of increase in the number of patients having this surgery but also there are no data concerning the features of patients actually having this surgery in clinical practice. The information cannot be derived from the grouped data of retrospectively identified patients in follow-up studies. Furthermore, there are inherent reporting and publication biases making published data on outcomes from specialist institutions an unreliable source of information on practice nationally. The need for data on current practice led to the formation of Grupo Español de Cirugía Metástasis Pulmonares de Carcinoma Colo-Rectal (GECMP-CCR; the Spanish Group) under the auspices of Sociedad Española de Neumologia y Cirurgĭa Torácica (SEPAR) in order to obtain high quality data nationwide in a short and defined time window to document existing practice in a healthcare system.

\section{METHODS}

Study group

GECMP-CCR-SEPAR (the Spanish Group) was constituted in January 2008. The entry criterion for thoracic surgery departments to be members of the group was to have performed at least five procedures of lung metastasectomy for colorectal carcinoma annually during the period 2005-2007. Departments performing fewer operations were not included but for those included in the group on that criterion, data were accepted whether or not that rate was maintained.

\section{Database}

The database created in discussion with the group is composed of over 160 elements in five groups:

1. Primary colorectal cancer characteristics comprising 33 elements.

2. Time elapsed from primary resection to the first lung metastasectomy comprising 17 elements.

3. Lung metastasectomy episodes, up to a maximum of five episodes with 80 elements each.

4. Relapse following each lung metastasectomy: 30 variables for each episode.

5. Study response variables including losses to follow-up (details available as a web extra).

Publications commonly refer to the disease-free interval or 'DFI'. The patient is by definition not 'disease free' and the data of first evidence of recurrence are unreliable. What is calculated in this paper (and is commonly given as DFI) is the interval between the primary operation and the pulmonary metastasectomy, referred to in this paper as the interoperative interval.

\section{Patient inclusion criteria}

Data were collected on patients who had a first lung metastasectomy operation between 1 March 2008 and 28 February 2010. Lung resection had to be performed with radical intent (that means complete resection of each metastasis) and there had to be no evident residual malignant disease in the lung at the end of the surgical procedure. There had to be histological confirmation that at least one of the lesions excised was consistent with lung metastasis from colorectal carcinoma. The presence of microscopic residual disease, as indicated by positive margins, was not a reason for exclusion. Microscopically detected cancer at the resection margin was registered as R1 disease, regardless of whether it involved parenchymal, vascular or bronchial margins.

In some patients, it was the resection of a lung metastasis, as a diagnostic procedure, which led to the diagnosis and treatment of colorectal cancer with curative intent. These patients are included in the database. Patients were not included if the resection of the lung nodule was a purely diagnostic procedure and no curative colorectal surgery was performed or if there was an occult primary which remained so until the end of the recruitment period.

\section{Preoperative detection of lymph node involvement}

No requirement was placed on the responding units to conform to a protocol for lymph node staging, but where done, positron emission tomography (PET) and PET/CT findings were recorded.

\section{Lymphadenectomy and lymph node involvement}

A common nomenclature was used for intraoperative lymphadenectomy techniques: (1) systematic lymph node dissection (including lobe-specific lymph node dissection) according to the definition of the Bronchogenic Carcinoma Cooperative Spanish Group; (2) systematic sampling: according to the definition of the Bronchogenic Carcinoma Cooperative Spanish Group and (3) minor lymphadenectomies: any other selective lymphadenectomy.

To be considered Stage N0p, there had to be at least systematic sampling or the disease was stage Nxp.

\section{Data on associated liver metastases and their treatment}

Associated liver metastases were recorded at three time points: as being present at the time of the primary resection, appearing after the primary resection or being present at the time of the pulmonary metastasectomy. In each case, the number treated was recorded.

\section{Analysis}

Descriptive analyses of patient demography, primary colorectal cancer and the metastatic disease for the total patient population were carried out. The analysts (FF and TT) relied on the centres' internal quality control for the veracity of all entries related to imaging and pathological diagnosis. Missing, outlying or implausible data were queried through direct enquiry with the data collectors at the various centres (RE). If items were missing, we retained the data available and stated the diminished denominator for that analysis. Simple 
descriptive statistical analysis was carried out looking at the mean, median, CIs and centiles according to the nature of the data and their distribution. The data report practice: there was no plan to draw any statistical inferences. The only subgroup analysis performed in this phase was by centre and this is for descriptive purposes only.

\section{RESULTS}

\section{Centres and patients}

Of the 35 centres that enrolled to submit data, 32 provided data for inclusion in the Spanish registry. There are 543 records in the database. The accrual of patients per centre is shown in figure 2. There were 6-43 patients per centre (median 16; IQR 10-23; N=543). There were $65 \%$ men and $35 \%$ women $(\mathrm{N}=541$; figure 3$)$. The mean age at the time of pulmonary metastasectomy was 65 years (range $32-88$ years, SD 10.2, N=529). The age distributions, centre by centre, are displayed in figure 4 .

\section{Site of primary colorectal cancer}

The site of the primary is shown in figure 5 for 526 tumours where a single location was recorded. In seven cases, non-adjacent colorectal sites were recorded, suggesting multiple primaries.

\section{Number of metastases removed}

Pulmonary metastases removed (1, 2, 3 and 4+) are shown centre by centre in figure 6. For 532 patients for whom the data are available, $293(55 \%)$ had a solitary metastasectomy; 104 (19.5\%) had two, 67 (12.6\%) had three and $68(12.9 \%)$ had four or more metastases resected. The frequency distribution of the number of metastases per operation shows the preponderance of solitary and low number metastases (figure 7).

\section{Interoperative interval}

The interval between resection of the primary and the metastasectomy is shown in figure 8. Based on data from 524 patients, the median interval was 28 months (IQR 16-43 months). In $17 \%$ (87 patients) the interval was under 12 months, in $47 \%$ (247) it was 12-36 months and in $36 \%$ (190 patients) it was over 36 months $(\mathrm{N}=524)$.

\section{Carcinoembryonic antigen status}

Carcinoembryonic antigen (CEA) at the times of assessment for metastasectomy was recorded in 414 patients. Among these, the median CEA was 2.72 (IQR 1.42-7). The distributions of the measurements by centre are shown in figure 9 . Of the 414 patients, $125(30 \%)$ had CEA assays above $5 \mathrm{ng} / \mathrm{ml}$ and 67/414 (16\%) had CEA greater than $10 \mathrm{ng} / \mathrm{ml}$.

Lung metastasectomy after previous liver metastasectomy In summary, 155 patients (29\%) had one or more liver metastases at some time prior to pulmonary metastasectomy. For 32 units, the liver metastasis rate ranged

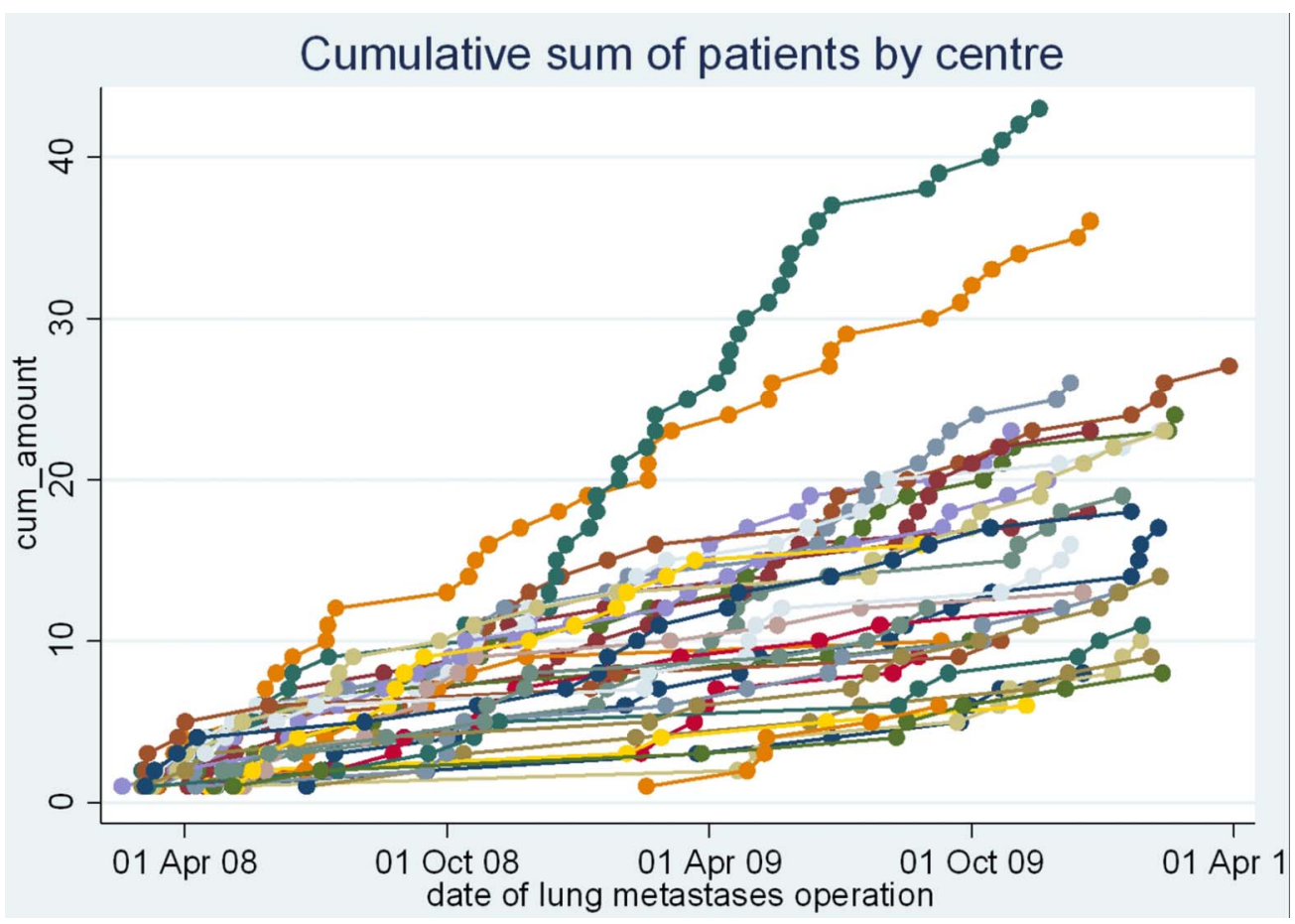

Figure 2 Accrual patterns for patients in the study. A few centres entered the study after 1 March 2008 to the end of February 2010. Data accrual for the late joiners appears to be consistent within the time frame of submission and there is no reason to think it was not a representative picture in practice. 


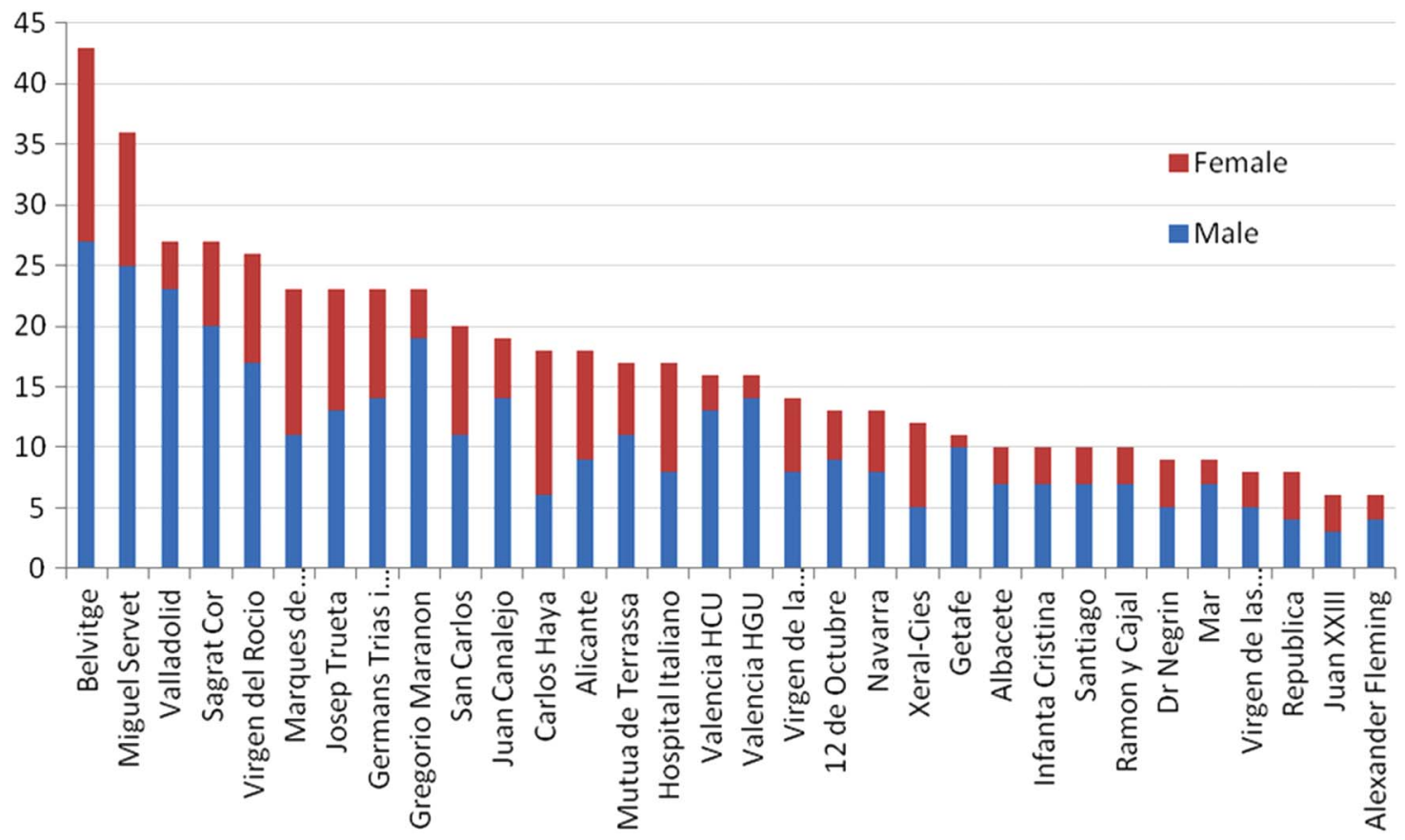

Figure 3 The number of cases per unit shown as men (blue) and women (red).

from $0 \%$ to $56 \%$ (median $25 \%$; IQR $19-33 \%$ ). In more detail, with respect to the time of liver metastases, of the 543 patients, $79(14.5 \%)$ had liver metastases at the time of primary colorectal resection, $104(19.2 \%)$ had metastases which appeared during the interoperative interval and $45(8.3 \%)$ at the time of pulmonary metastasectomy. Figure 10 shows the variation by unit in the presence of metastases at each of these times. The proportions treated at each of those times were $14 \%, 19 \%$ and $7.7 \%$, respectively. These counts are not mutually exclusive and an individual patient could feature in one, two or all three counts because the appearance, evaluation and treatment of metastases is a dynamic process.

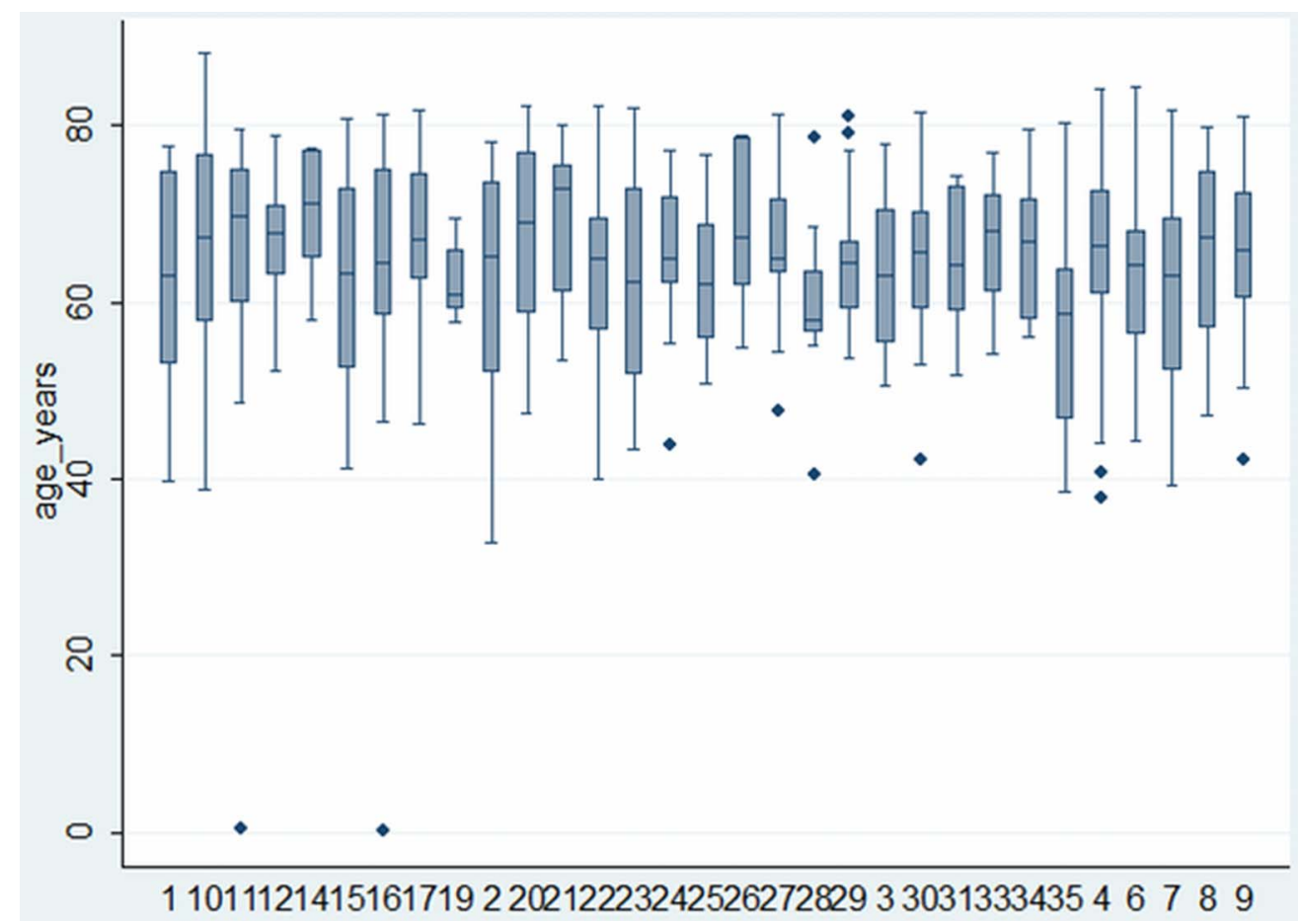

Figure 4 The age distribution for each centre showing the median, IQR, 10th and 90th centiles and extreme values. 


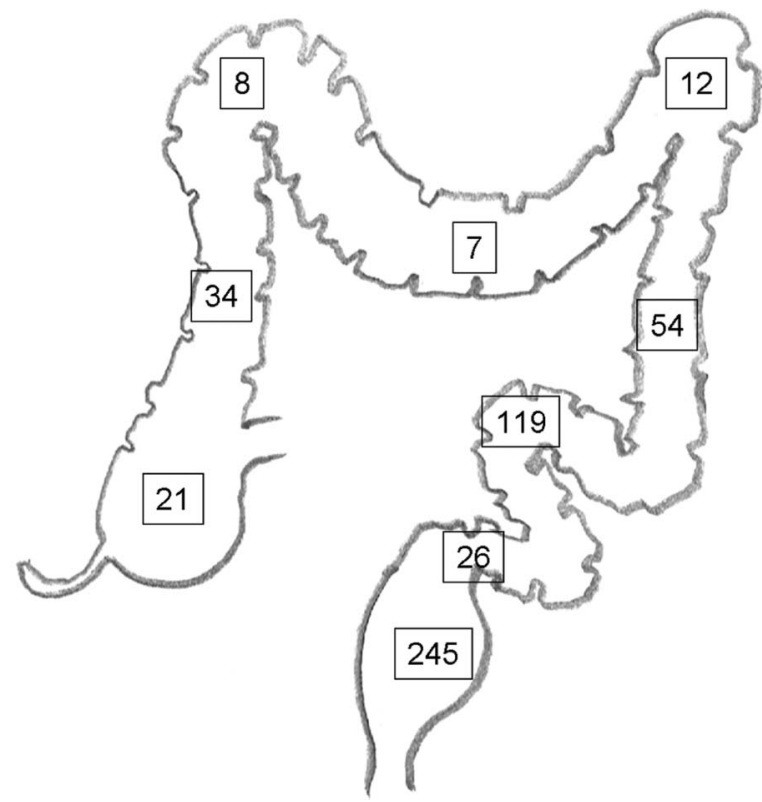

Figure 5 Location of the primary colon and rectal tumours. About $46 \%$ of the metastatic were from the rectum while usually about a third of the primary colorectal cancers are in the rectum. This excess is maybe because the systemic venous drainage of the rectum is more likely to allow lung only or lung first metastases while colon cancer may metastasise selectively to the liver via the hepatic portal vein. This simplistic anatomical explanation is disputed, but there are data to support the differential lung/liver metastasis predilection. $^{24}$

\section{Completeness of resection}

In $95 \%$ of patients, there was $\mathrm{R} 0$ resection (no cancer at the resection margin at microscopy). The $\mathrm{R} 1$ resection rates by unit ranged from $0 \%$ to $22 \%$ (median $0 ; \mathrm{IQR}$ $0-7 \%)$. It should be noted that these rates are unstable. The actual numbers for the unit with the highest rate were two R1 resections among nine patients for a rate of $22 \%$ (95\% Confidence Limits 3-60\%). More or fewer R1 resections would radically alter ranking when there are numbers both of events and denominators.

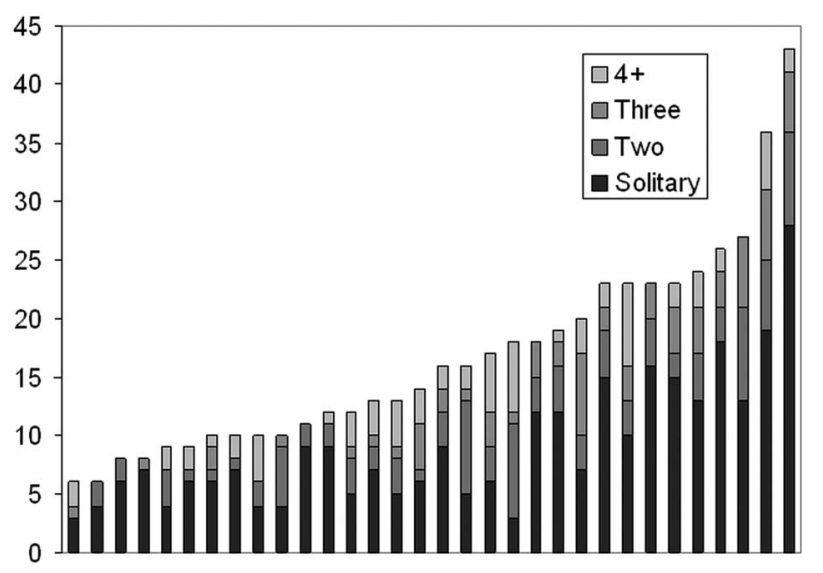

Figure 6 The centre-by-centre number of metastases resected in individual cases.
Mediastinal staging prior to pulmonary metastasectomy PET data for mediastinal nodes were recorded in 388/ $543(71.4 \%)$ patients. Of these 388 , the stages were N0 in $357(92 \%), \mathrm{N} 1$ in $7(1.8 \%)$, N2 in $15(3.9 \%)$ and N3 in $9(2.3 \%)$.

\section{Lymphadenectomy}

The overall proportion of patients among the 543 who underwent lymphadenectomy according to the definitions included systematic lymph node dissection (9\%), systematic sampling (18\%) and minor lymphadenectomy $(19 \%)$. The patients having any form of lymphadenectomy varied by centre from $9 \%$ to $100 \%$ (median $40 \%$; IQR 29-60\%). These are mutually exclusive and are shown in figure 11 with the contribution of the three types indicated within the total proportion having any form of lymphadenectomy.

\section{DISCUSSION}

The Spanish Group's registry represents the largest data set of prospectively collected cases of pulmonary metastasectomy for colorectal cancer known to us. Contemporary patient-level data are not available from any other published source. The International Registry of Lung Metastasectomy published 15 years ago did not separately identify colorectal cancer metastases. ${ }^{12}$ Systematic reviews, such as those from Pfannschmidt et $a \bar{l}^{\tilde{6}}$ and Gonzalez $e t a l^{3}$ and the most inclusive, a quantitative synthesis of 51 papers summarising data on 3504 patients, ${ }^{4}$ are properly regarded as secondary sources because they rely on amalgamation of already grouped data. The largest primary data source of which we are aware is the combined series from Duke and the Memorial Sloan-Kettering Cancer Centre comprising 378 patients over a 10-year period from 1998 to 2007 and published in 2009. ${ }^{13}$

By contrast with these retrospectively collected data sets, the Spanish registry patients were identified prospectively and collected from as many centres as possible within a short time frame of 2 years. The 32 Spanish hospitals contributing to the study take care of 28 million people, around $60 \%$ of the Spanish population (46.6 million in 2009). Based on the work of the ESTS Lung Metastasectomy Project ${ }^{10}$ and the many reports of individual series from many European countries, these data are believed to be representative of current European practice.

Case selection in the Spanish registry and published cohorts versus surgeon reported beliefs

The most striking finding is the contrast between this 2-year snapshot of practice and what thoracic surgeons responding to the ESTS survey thought to be their practice. ${ }^{9}$ The reality is much more selective and conservative than believed by surgeons responding to the ESTS survey, with respect to the number of metastases removed and the interval since resection of the primary. 


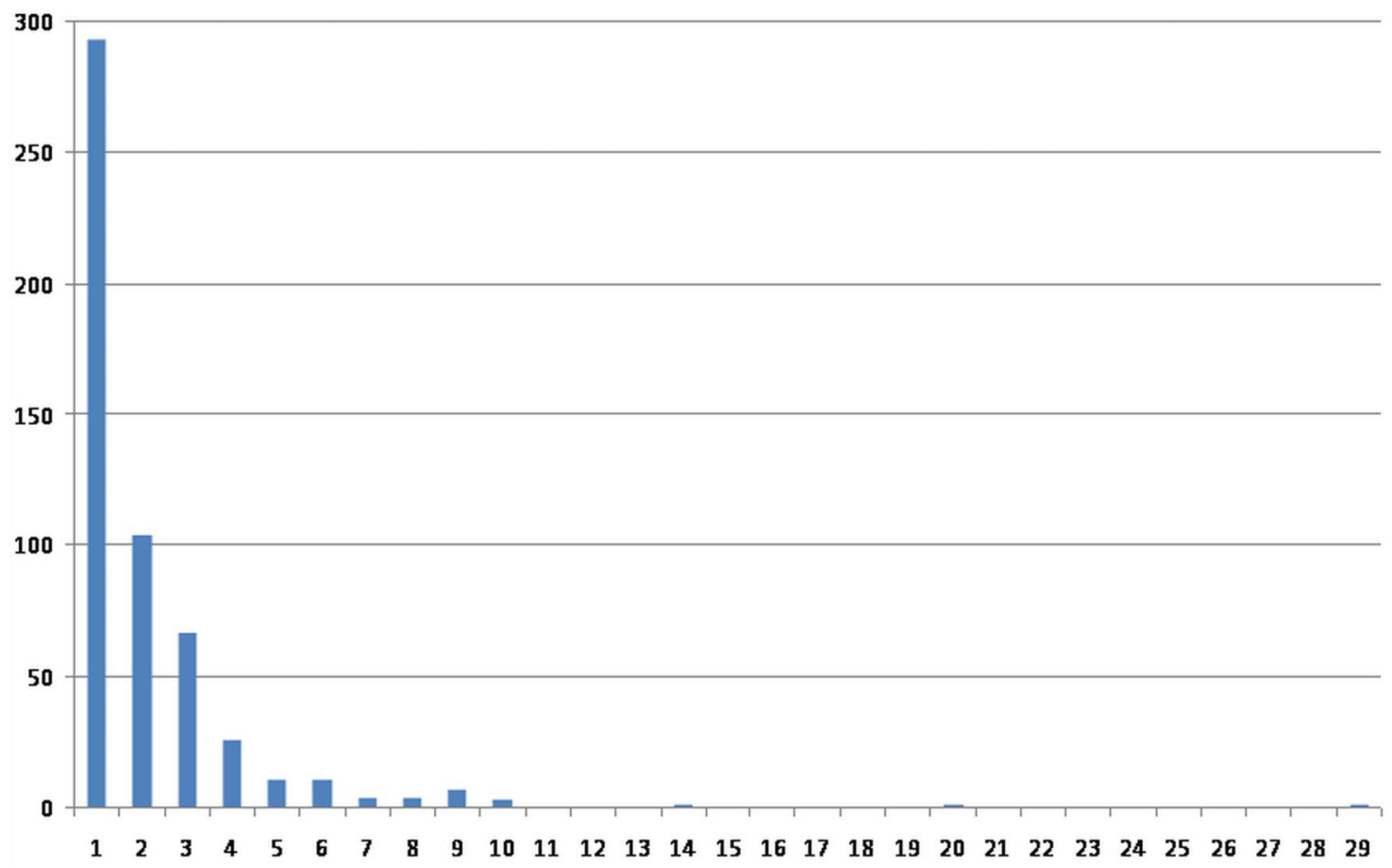

Figure 7 Frequency distribution of the metastatic number.

Quantitative synthesis of the historical data in the systematic reviews shows the practice, as reported in surgical follow-up studies, to be dominated by patients with fewer metastases, and a longer interoperative interval and non-elevated CEA. ${ }^{4}$ These factors are known from repeated multivariate analyses to be associated with better 5-year survival rates after metastasectomy. ${ }^{3} 7$ It should not be overlooked that these factors are also those associated with a more favourable natural history.

Some characteristics of the patients in the Spanish group's registry are shown in table 1 , in comparison to the largest known aggregation of data that we are aware

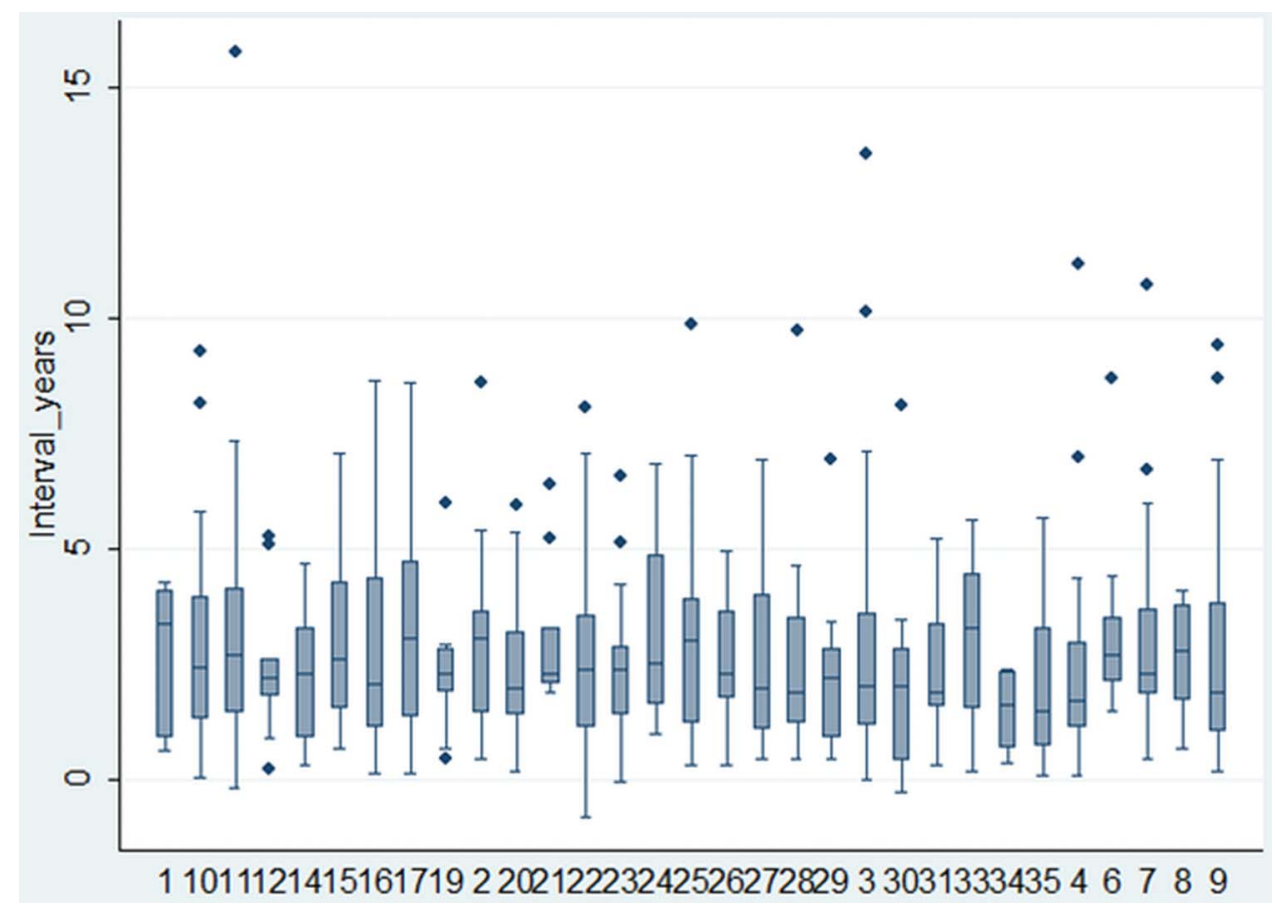

Figure 8 The interval between primary resection and pulmonary metastasectomy: median, IQR, 10th and 90th centiles and outliers. 


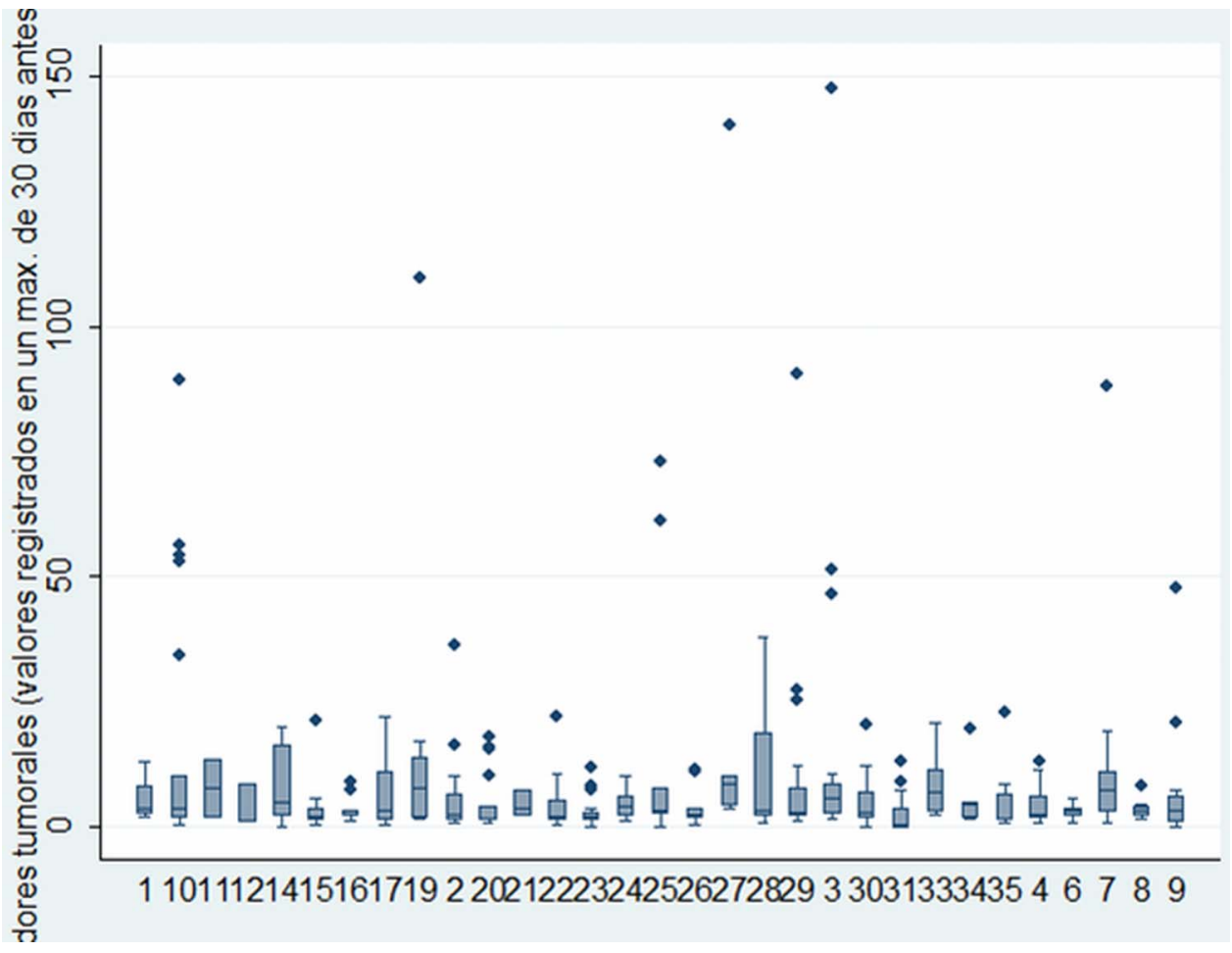

Figure 9 Carcinoembryonic antigen by centre: median, IQR, 10th and 90th centiles and outliers.

of, from the systematic review and quantitative synthesis ${ }^{4}$ and the large Duke/MSK-CC. ${ }^{13}$ The time frame of the former is wide, going back to the 1960s, while the latter is more constrained (1998-2007), but the data are broadly similar. The 5-year increase in average age of the patients is as might be expected when recent practice is compared with that of even a few years ago. The Spanish data are comparable with observational data from other sources, and therefore it is the survey which is misleading. ${ }^{9}$ Surgeons' recollections of what they do are not necessarily in line with the documented record of practice.

\section{Number of metastases and the interval between primary surgery and metastasectomy}

The two most consistently reported factors related to survival are the number of metastases and the interval between resection of the primary cancer and metastasectomy. There has been a tendency to allow optimism to expand the criteria for metastasectomy. Although there have been many publications exploring favourable and unfavourable factors for survival, authors have only recently explicitly stated groups of patients who gain no survival benefit. Onaitis et $a l^{13}$ (Duke and MSK-CC) were unusual in this regard and stated, "Medical management alone should be considered standard for patients who have both three or more pulmonary metastases and less than 1 year DFI." The Spanish registry data for number and interval are plotted in figure 12 demarcating patients above and to the right of these red lines. The latest meta-analysis derived an HR for poor outcome which doubles for multiple metastases (HR 2.04, 95\% CI 1.72 to 2.41 ) and multiple means simply more than one ${ }^{3}$ concurring with the findings of an earlier study in which the more favourable outcomes were in patients with no more than one metastatic lesion ${ }^{7}$ (marked in amber in figure 12). Nevertheless, the authors conclude, 'it seems currently unfair to deny surgery for those patients with two to four lesions'. ${ }^{3}$ This value laden statement depends on what is meant by 'unfair'. Some might think it unfair to be put through surgery when benefit is improbable.

There are obvious technical considerations in preferring to offer an intervention to patients with fewer metastases. A proposal has been made to regard the existence of a few metastases as an entity, the oligometastatic state ${ }^{1415}$ set at five or fewer by its protagonists. The sobriquet has been applied to colorectal cancer, ${ }^{7}$ but it may be more plausible to regard the fewness of metastases as nothing other than the tail of a skewed distribution in which the fewer metastases are simply a marker for less aggressive cancer. However, the fewer metastases there are, the more interesting they are to practitioners. Oligometastasis represent a therapeutic opportunity not only for surgery but also for radiofrequency ablation and the latest radiotherapy techniques $(\mathrm{SBRT}){ }^{16}$ with which there is an upper limit in terms of practicalities to how many metastases can be removed. In contrast, but unrepresentative of practice, according to a report advocating laser as a tool to allow the lung parenchyma to be spared in open surgery, as many as 124 metastases were resected. ${ }^{17}$ In general, the fewer the 


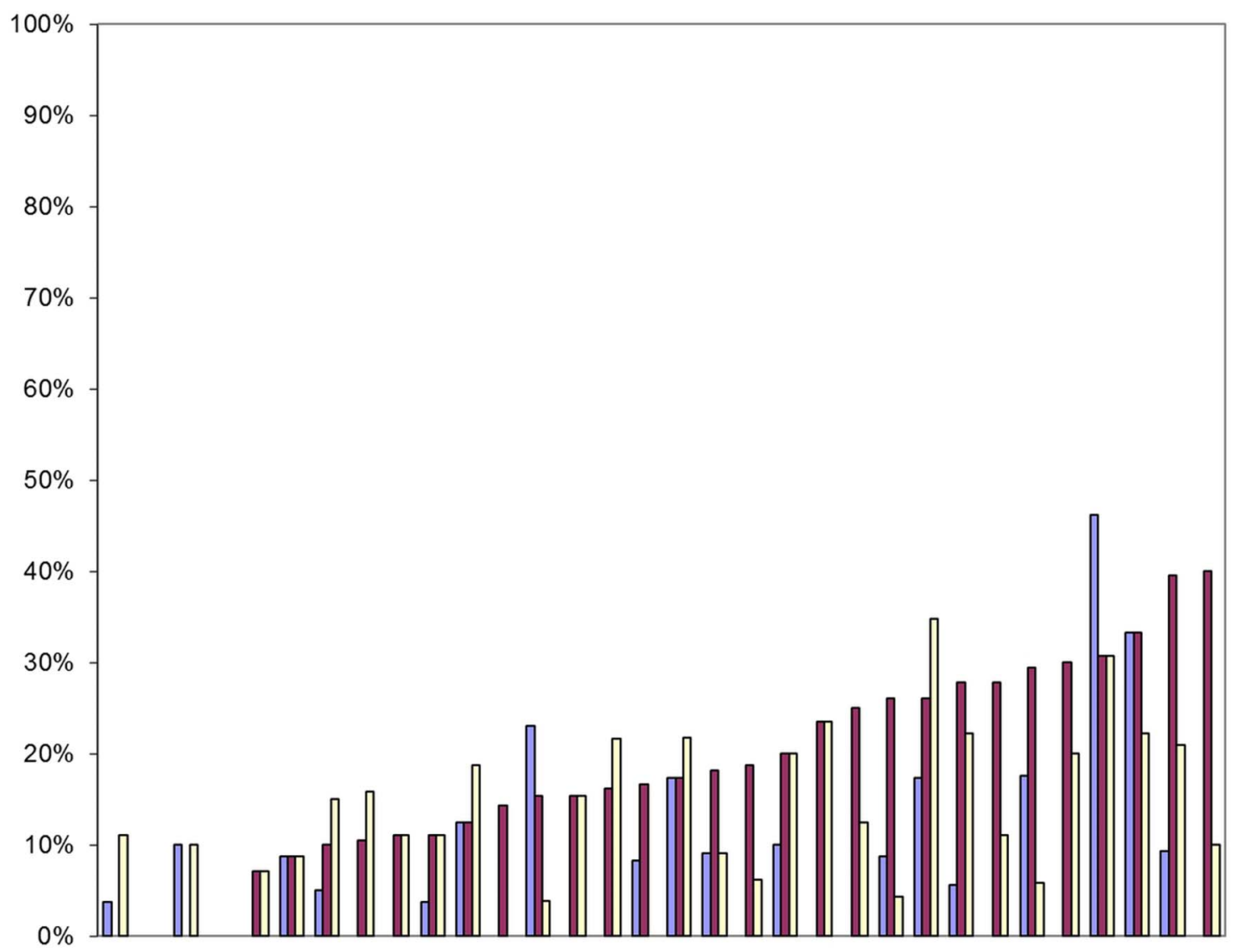

Figure 10 Proportion of patients in each centre who had liver metastases at the time of the primary resection (blue), appeared since the primary resection (maroon) or were present at the time of the pulmonary metastasectomy. For ease of reading, they are ranked by those appearing subsequent to primary resection, but the data are not aggregated because the counts are not mutually exclusive and there is a dynamic process of metastases appearing and being treated.

number of metastases, the more likely is metastasectomy to be offered.

For the interoperative interval, the clinical incentives work in the opposite direction. The effect can be seen in figure 12 where surgeons select patients with fewer metastases but evidently are not able to restrict surgery to those with longer intervals. There can be no absolute threshold proposed along the time continuum but indicative intervals, below which long survival cannot be anticipated, are variably suggested at 12 months, ${ }^{13}$ in the range of $19-39$ months $^{3}$ and 36 months $^{7}$ in recent pooled analyses. Interpretation is made more difficult

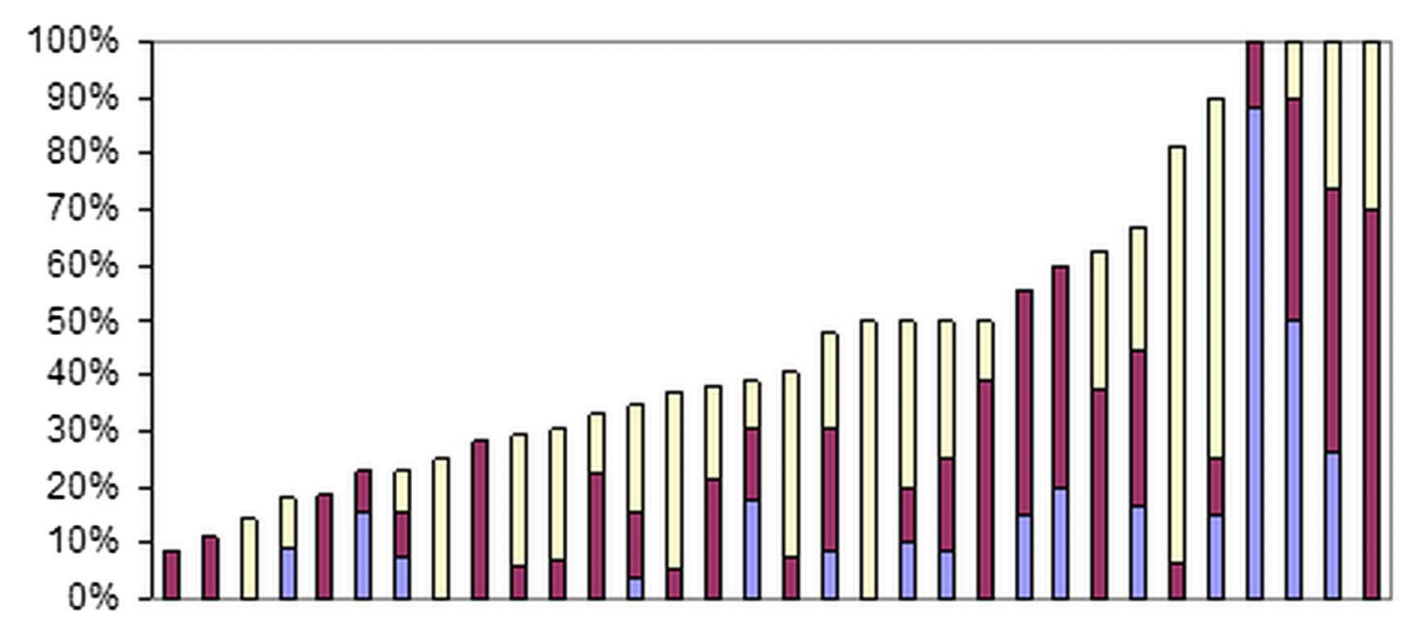

Figure 11 Proportion of patients having any lymphadenectomy in each centre, ranked by overall number with systematic lymph node dissection in blue, systematic sampling in maroon and minor lymphadenectomies in yellow. 
Table 1 Basic elements of the data included in the Spanish Registry (GECMP-CCR-SEPAR) are compared with those in the most comprehensive systematic review ${ }^{4}$ of 51 retrospective studies and the combined Duke and MSK-CC follow up study ${ }^{13}$

\begin{tabular}{llll}
\hline & $\begin{array}{c}\text { GECMP } \\
\mathbf{N = 5 4 3 *}\end{array}$ & $\begin{array}{l}\text { Systematic } \\
\text { review } \\
\mathbf{N = 2 5 0 4}\end{array}$ & $\begin{array}{l}\text { Onaitis }^{\mathbf{1 3}} \\
\mathbf{N = 3 7 8}\end{array}$ \\
\hline Mean age (years) & 65 & 60 & 61 \\
Sex (\%male) & 65 & 60 & 60 \\
Solitary metastases & 55 & 60 & 60 \\
Median interval & 28 & 36 & 24 \\
since primary & & & \\
resection (months) & & & \\
\hline
\end{tabular}

Given the very large time scale of about 50 years of practice represented altogether, the case selection is remarkably similar. *Some data are missing from the Spanish registry. The denominators are 529 for age, 541 for sex, 532 for the count of metastases and 524 for the interoperative interval. The percentage is of those where data are available. GECMP, Grupo Español de Cirugía Metástasis Pulmonares.

because not all studies use the same definition of DFI. Some authors use the interoperative interval as a surrogate for the DFI (as we have carried out here), but others used the exact interval from primary colorectal surgery to the time when the metastatic lesions were first detected following that primary surgery. ${ }^{7}$ Gonzalez $e t a \hat{l}^{3}$ remarked on the problem of meta-analysis when authors provided the information in different ways. Common reporting standards would certainly make analytical work easier. ${ }^{18}$ But however it is defined, if the metastases are detected early, a deliberate policy of waiting to ensure that these are not the few of many more to come must be unbearable for many patients, set against a hope for cure and the sense of a missed opportunity. The perception that there is any advantage in haste is not based on the evidence that already exists in observational studies.

\section{Carcinoembryonic antigen}

Of the patients in the Spanish registry in whom CEA was measured, it was elevated (CEA $>5 \mathrm{ng} / \mathrm{ml}$ ) in $30 \%$. During the 1980s, a CEA assay was proposed to trigger asecond-look laparotomy including inspecting the liver with a view to resection. A randomised trial found that this increased the number of operations but not the survival of the patients. ${ }^{19} 20$ Some oncologists continue to use a CEA assay as a means of surveillance to trigger further imaging and if lung metastases are discovered, this may lead to a referral to a thoracic surgeon for their removal. ${ }^{21}$ A thoracic surgeon, aware of the evidence, would rightly be inclined to advise against surgery because elevated CEA is a marker for a poor outcome. ${ }^{346}$ This sends mixed messages and the risk of confusion in the minds of patients. ${ }^{22}$

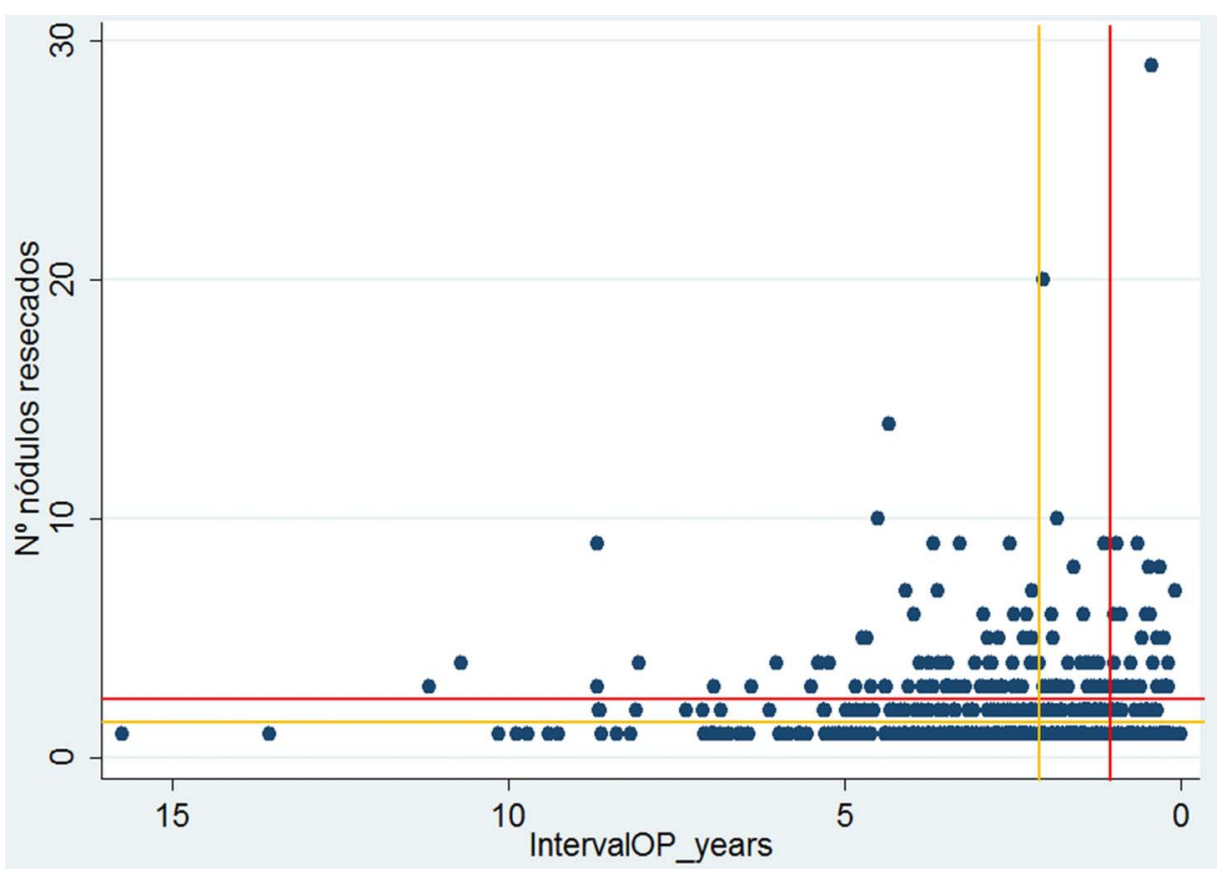

Figure 12 Scatterplot of the interoperative interval and number of metastases. The red lines demarcate the thresholds (an interval of less than 12 months and 3 or more metastases) beyond which outcomes were always poor in the Duke/MSK-CC experience. ${ }^{13}$ The amber lines demarcate the thresholds in most of the meta-analyses by Gonzales et al, ${ }^{3}$ again indicating the patients likely to recur above and to the right of the pair of lines. The factors which are favourable for survival (solitary metastasis and long interval) are to the bottom left of the graph where one might anticipate the concentration of patients to be. It appears that selecting patients with fewer metastases occurs more naturally than deliberately delaying surgery for the selected few with longer intervals. 


\section{Lung and liver metastasectomy}

The practice of hepatic resection of colorectal liver metastases, in selected patients, was adopted internationally by the late $1990 \mathrm{~s}^{23}$ The liver is the commonest site of metastases and the route of spread is via the liver, which explains the excess of lung first or only metastases with rectal cancer compared with colon cancer. ${ }^{21}{ }^{24} \mathrm{An}$ innovative analysis from the Cleveland Clinic of patients who have had hepatic resection for metastases, and subsequently were found to have lung metastases, was used to argue in favour of resection of both, but in very highly selected patients. ${ }^{25}$ However, the shape of the survival curve is unlike that of usual cancer survival because the performance of the second operation (in whichever order) is conditional on having survived and recovered from the first to be considered for the second. Recent European studies report that patients who have had hepatic resection are increasingly being referred for pulmonary metastasectomy. The series are small with $17 / 84^{26}$ and $27 / 70^{27}$ of the patients having pulmonary metastases having had prior liver resection (about 20\% and $40 \%$ ). Of the patients in the Spanish registry, based on much larger numbers, 29\% (155/543) had prior liver metastasectomy. The wide variation among centres $(0-56 \%)$ is likely to reflect referral patterns. Thoracic surgeons working in a hospital with liver surgery are more likely to be referred patients from other centres not performing liver resections. With respect to survival for those with liver and lung resection, of note are the final words of the larger of these series: "prolonged disease-free survival remains the exception, and seems to occur only in patients with a single lung lesion." 27

\section{Degree of selection and its effect}

The starting point for cases in the Spanish registry is that they had a proven metastasis resected. The population denominator from which these patients were drawn is not known, and nor is the number of patients considered for metastasectomy, or the number of patients who had a nodule removed which proved not to be a metastasis. This is a limitation in common with most clinical reports, nearly all of which are follow-up studies.

Spanish cancer registry data allow an estimate of the proportion of patients with colorectal cancer who feature in the Spanish metastasectomy registry. The incidence of colorectal cancer in the Spanish population is of the order of 45 men and 35 women $/ 100000 \cdot{ }^{28}$ Based on an estimated population base of 28 million, the number of patients with colorectal cancer in a 2-year period would have been around 22400 cases of whom about half might have had metastatic disease. Based on these estimates, the patients in the dataset might represent about 5\% of patients with metastatic colorectal cancer or $2.5 \%$ of all patients with colorectal cancer.

A report from Turin from three hospital databases identified 1411 patients with metastatic colorectal cancer recorded between 1994 and $2010 .{ }^{29}$ Of them, 50 patients $(3.5 \%)$ were selected to have pulmonary metastasectomy.
In a Japanese study of patients who had an R0 curative resection of colorectal cancer from 2000 to 2006, 18/764 (2.4\%) were selected for pulmonary metastasectomy. ${ }^{21}$ We can say no more than that the selected proportion of patients in the Spanish registry is of a similar order of magnitude to that in other reports where some estimate of the denominator is available. What is consistent is that only 1 in 30-40 patients with advanced colorectal cancer have pulmonary metastasectomy. There remains the possibility that the subsequent survival of these patients owes more to selection of a subset of inherently more favourable cases than being attributable to the effect of the pulmonary metastasectomy. ${ }^{8} 30$

\section{Solitary nodules of unknown histology}

Patients who had an excision of a solitary nodule, which proved not to be a colorectal cancer metastasis, are not recorded in the Spanish registry. The colorectal surveillance pathway overlaps with the clinical pathway for patients who present with a solitary pulmonary nodule. ${ }^{31}$ The research question in the present study is thus narrowed to only patients in whom the nodule proved to be a colorectal cancer metastasis. There have to be boundaries to a data retrieval exercise, but this post hoc entry criterion should be remembered if these data are used outside their intended purpose.

\section{Case for a randomised control trial}

The leaders of the ESTS Working Group concluded, "In the absence of a randomized controlled trial looking at the effectiveness of pulmonary metastasectomy on survival and quality of life, it is unlikely that the current practice will ever be influenced." The randomised trial that emanated from the working group's deliberations (PulMiCC) $^{32} 33$ is open internationally. The Spanish population-based study cannot answer questions about effectiveness in any patient group but does provide information about practice against which the generalisability of a randomised trial can be tested.

\section{Author affiliations}

${ }^{1}$ Department of General Thoracic Surgery, Miguel Servet University Hospital, Zaragoza, Spain

${ }^{2}$ Department of Cardiothoracic Surgery, NHLI, Imperial College London, London, UK

${ }^{3}$ Clinical Operational Research Unit (CORU) University College London, London, UK

${ }^{4}$ Department of General Thoracic Surgery, Sagrat Cor University Hospital, Barcelona, Spain

Contributors RE, JJR and LM instigated the study on behalf of Grupo Español de Cirugía Metástasis Pulmonares de Carcinoma Colo-Rectal (GECMP-CCR) de la Sociedad Española de Neumologĭa y Cirurgía Torácica (SEPAR). TT agreed to analyse the data with Francesca Fiorentino. TT wrote the first draft and final version of the manuscript and edited all versions of the manuscript. FF did the technical analysis and contributed to the drafting and figures. All authors have contributed to the revision of the manuscript and agree on the final version.

Funding GECMP-CCR-SEPAR Register was funded by Ethicon-Endosurgery. Competing interests None. 
Ethics approval Comité Etico de Investigación Clínica de Aragón (CEICA). Provenance and peer review Not commissioned; externally peer reviewed.

Data sharing statement The complete data set can be made available for further analysis on application to GECMP.

\section{REFERENCES}

1. NICE. Guidance on Cancer Services. Improving outcomes in colorectal cancer. NICE May 2004 Manual update. 2004. http://www. nice.org.uk/page.aspx?o=CSGCCfullguideline

2. Poston G, Guideline Development Group. Colorectal cancer: the diagnosis and management of colorectal cancer. National Institute for Health and Clinical Excellence. 2011. http://guidance.nice.org.uk/ CG131/Guidance/pdf/English (accessed 7 Oct 2012).

3. Gonzalez M, Poncet A, Combescure C, et al. Risk factors for survival after lung metastasectomy in colorectal cancer patients: a systematic review and meta-analysis. Ann Surg Oncol 2013;20:572-9.

4. Fiorentino F, Hunt I, Teoh $\mathrm{K}$, et al. Pulmonary metastasectomy in colorectal cancer: a systematic review and quantitative synthesis. $J$ R Soc Med 2010;103:60-6.

5. Pfannschmidt J, Dienemann H, Hoffmann H. Surgical resection of pulmonary metastases from colorectal cancer: a systematic review of published series. Ann Thorac Surg 2007;84:324-38.

6. Pfannschmidt J, Hoffmann H, Dienemann H. Reported outcome factors for pulmonary resection in metastatic colorectal cancer. $J$ Thorac Oncol 2010;5(6 Suppl 2):S172-8.

7. Salah S, Watanabe K, Welter S, et al. Colorectal cancer pulmonary oligometastases: pooled analysis and construction of a clinical lung metastasectomy prognostic model. Ann Oncol 2012;23:2649-55.

8. Aberg T, Malmberg KA, Nilsson B, et al. The effect of metastasectomy: fact or fiction? Ann Thorac Surg 1980;30:378-84.

9. Internullo E, Cassivi SD, Van Raemdonck D, et al. Pulmonary metastasectomy: a survey of current practice amongst members of the European Society of Thoracic Surgeons. J Thorac Oncol 2008;3:1257-66.

10. Van Raemdonck D, Friedel G. The European Society of Thoracic Surgeons Lung Metastasectomy Project. J Thorac Oncol 2010;5(6 Suppl 2):S127-9.

11. Treasure $T$, Fallowfield L, Lees $B$, et al. Pulmonary metastasectomy in colorectal cancer: the PulMiCC trial. Thorax 2012;67:185-7.

12. Pastorino $U$, Buyse $M$, Friedel $G$, et al. Long-term results of lung metastasectomy: prognostic analyses based on 5206 cases. $J$ Thorac Cardiovasc Surg 1997;113:37-49.

13. Onaitis MW, Petersen RP, Haney JC, et al. Prognostic factors for recurrence after pulmonary resection of colorectal cancer metastases. Ann Thorac Surg 2009;87:1684-8.

14. Hellman S, Weichselbaum RR. Oligometastases. J Clin Oncol 1995;13:8-10.

15. Weichselbaum RR, Hellman S. Oligometastases revisited. Nat Rev Clin Oncol 2011;8:378-82.

16. Treasure T. Oligometastatic cancer: an entity, a useful concept, or a therapeutic opportunity? J $R$ Soc Med 2012;105:242-6.

17. Rolle A, Pereszlenyi A, Koch $R$, et al. Is surgery for multiple lung metastases reasonable? A total of 328 consecutive patients with multiple-laser metastasectomies with a new 1318-nm Nd:YAG laser. $J$ Thorac Cardiovasc Surg 2006;131:1236-42.

18. Fiorentino F, Treasure T. A plea for consistency in the reporting of surgical series illustrated with an analysis of 51 follow-up reports of pulmonary metastasectomy in colorectal carcinoma. J Thorac Oncol 2010;5(6 Suppl 2):S192-5.

19. Northover J, Houghton J, Lennon T. CEA to detect recurrence of colon cancer. JAMA 1994;272:31.

20. Northover J. The use of prognostic markers in surgery for colorectal cancer. Eur J Cancer 1995;31A:1207-9.

21. Watanabe K, Saito N, Sugito M, et al. Incidence and predictive factors for pulmonary metastases after curative resection of colon cancer. Ann Surg Oncol 2013;20:1374-80.

22. Treasure T. Carcinoembryonic antigen: its place in decision making for pulmonary metastasectomy in colorectal cancer. J Thorac Oncol 2010;5(6 Suppl 2):S179-81.

23. Grunhagen D, Jones RP, Treasure T, et al. The history of adoption of hepatic resection for metastatic colorectal cancer: 1984-95. Crit Rev Oncol Haematol, 2013;86:222-31.

24. Tan KK, Lopes GL Jr, Sim R. How uncommon are isolated lung metastases in colorectal cancer? A review from database of 754 patients over 4 years. J Gastrointest Surg 2009;13:642-8.
25. Robinson BJ, Rice TW, Strong SA, et al. Is resection of pulmonary and hepatic metastases warranted in patients with colorectal cancer? J Thorac Cardiovasc Surg 1999;117:66-75.

26. Zabaleta J, Aguinagalde B, Fuentes MG, et al. Survival after lung metastasectomy for colorectal cancer: importance of previous liver metastasis as a prognostic factor. Eur J Surg Oncol 2011;37:786-90.

27. Gonzalez M, Robert JH, Halkic N, et al. Survival after lung metastasectomy in colorectal cancer patients with previously resected liver metastases. World J Surg 2012;36:386-91.

28. Lopez-Abente G, Ardanaz E, Torrella-Ramos A, et al. Changes in colorectal cancer incidence and mortality trends in Spain. Ann Oncol 2010;21(Suppl 3):iii76-82.

29. Tampellini M, Ottone A, Bellini $E$, et al. The role of lung metastasis resection in improving outcome of colorectal cancer patients: results from a large retrospective study. Oncologist 2012;17:1430-8.

30. Utley M, Treasure T. Interpreting data from surgical follow-up studies: the role of modeling. J Thorac Oncol 2010;5(6 Suppl 2):S200-2.

31. Gould MK, Fletcher J, lannettoni MD, et al. Evaluation of patients with pulmonary nodules: when is it lung cancer? ACCP evidence-based clinical practice guidelines (2nd edition). Chest 2007;132(3 Suppl):108S-30S.

32. Treasure T, Fallowfield L, Lees B. Pulmonary metastasectomy in colorectal cancer: the PulMiCC trial. J Thorac Oncol 2010;5(6 Supp 2):S203-6.

33. Treasure T, Lees B, Russell C. Pulmonary metastasectomy in colorectal cancer: recruitment in the PulMiCC trial. Eur J Surg Oncol 2012;38:1130. Abstract

\section{APPENDIX}

Members of the Spanish Group for Surgery of Lung Metastases from Colorectal Carcinoma GECMP-CCR-SEPAR.

Coordinators:

Juan J. Rivas (Miguel Servet University Hospital, Zaragoza).

Laureano Molins (Sagrat Cor University Hospital, Barcelona).

Secretary:

Raul Embun (Miguel Servet University Hospital, Zaragoza).

Local heads and Departments:

Javier Ruiz (H.U Virgen de las Nieves, Granada).

Carlos Pagés (H.G.U Carlos Haya, Málaga).

Javier de la Cruz (H.U Virgen del Rocío, Sevilla).

Raul Embun (H.U Miguel Servet, Zaragoza).

Jorge Freixinet (H.U Dr. Negrín, Las Palmas).

Miguel Carbajo (H.U Marqués de Valdecilla, Santander).

Carlos Rombolá (H.G.U de Albacete)

Félix Heras (H.G.U de Valladolid).

José M. Mier (H.U Sagrat Cor, Barcelona).

Francisco Rivas (H.U de Bellvitge, Hospitallet de Llobregat, Barcelona)

Alberto Rodríguez (H.U. del Mar, Barcelona).

Emilio Canalís (H.U. Juan XXIII, Tarragona).

Matilde Rubio (H.U. Josep Trueta, Girona).

Sergi Call (H.U. Mutua de Terrassa, Barcelona).

Esther Fernández (H.U. Germans Trias i Pujol, Badalona, Barcelona).

Santiago Ga Barajas (H.U. Infanta Cristina, Badajoz).

José M. Ga Prim (H.C.U. de Santiago de Compostela, A Coruña).

Diego González (C.H.U. de A Coruña).

Montse Blanco (C.H.U. de Vigo, Pontevedra).

José Ramón Jarabo (H.C. San Carlos, Madrid).

Rafael Peñalver (H.U. Gregorio Marañón, Madrid).

Gemma Muñoz (H.U. Ramón y Cajal, Madrid).

Mª Carmen Marrón (H.U. 12 de Octubre, Madrid).

Beatriz de Olaiz (H.U. de Getafe, Madrid).

Andrés Arroyo (H.U. Virgen de la Arrixaca, Murcia).

Akiko Tamura (Clínica U. de Navarra, Pamplona).

Richard Wins (H.C.U. de Valencia).

Antonio Arnau (H.G.U. de Valencia).

Juan Manuel Córcoles (H.G.U. de Alicante).

Guillermo Carriquiry (H. Maciel, Universidad de la República, Montevideo, Uruguay).

Moisés Rosenberg (Instituto Oncológico Alexander Fleming,

Buenos Aires, Argentina).

David Smith (H. Italiano, Buenos Aires, Argentina). 